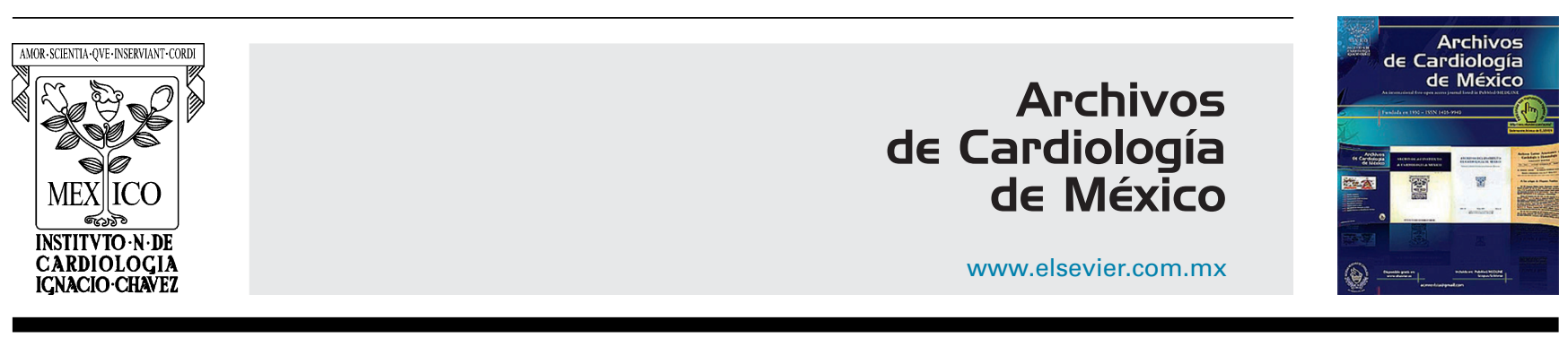

EDITORIAL

\title{
El valor clínico del examen físico en cardiología: revisión de la evidencia
}

\section{Clinical value of cardiovascular physical examination: A review of evidence}

\author{
Diego Araiza-Garaygordobil ${ }^{\mathrm{a}, *}$, Edgar Illescas-González ${ }^{\mathrm{a}}$, Jorge Eduardo Cossío-Aranda ${ }^{\mathrm{b}}$, \\ Jorge Kuri-Alfaro ${ }^{b}$ y José Fernando Guadalajara-Boo ${ }^{c}$
}

\author{
a Departamento de Cardiología, Instituto Nacional de Cardiología Ignacio Chávez, Ciudad de México, México \\ b Consulta externa, Instituto Nacional de Cardiología Ignacio Chávez, Ciudad de México, México \\ c Departamento de Enseñanza, Instituto Nacional de Cardiología Ignacio Chávez, Ciudad de México, México
}

Recibido el 19 de abril de 2017; aceptado el 6 de junio de 2017

El progreso de la medicina moderna y la cardiología se encuentra ligado de forma íntima a los avances en la tecnología biomédica. Hace apenas 50 años que el manejo de cualquier condición se basaba principalmente en los datos inferidos después de una historia clínica y un examen físico completos $^{1}$. La clínica como guía del ejercicio de la medicina ha sido desplazada por la tecnología médica moderna en todas las ramas de la medicina: en la actualidad, el estado del arte de la medicina se basa en datos precisos obtenidos mediante recursos tecnológicos muy avanzados, incluyendo medicina genómica, biomarcadores y métodos de imagen de la más alta gama.

El advenimiento de las tecnologías de bolsillo como el ultrasonido compacto y la mayor disponibilidad de recursos diagnósticos han llevado al clínico a enfrentarse a la controversia de si la tecnología desplazará completamente al examen físico. Este concepto es especialmente controversial en la comunidad de cardiólogos, donde la frase

\footnotetext{
* Autor para correspondencia. Calle Juan Badiano 1, Tlalpan, Belisario Dominguez Sección XVI, 14080 Ciudad de México, México.

Correo electrónico: dargaray@gmail.com

(D. Araiza-Garaygordobil).
}

«el ecocardiograma ha desplazado el estetoscopio» ha sido citada en varias ocasiones ${ }^{2,3}$.

Sin embargo, el examen físico sigue siendo un recurso útil, disponible a la cabecera del enfermo y que puede proveer una gran cantidad de datos significativos sin un costo económico añadido, especialmente si se reconocen sus ventajas y limitaciones. Esta revisión tiene como objetivo repasar la evidencia en relación con la eficacia del examen físico en algunas de las condiciones clínicas más comunes en cardiología (insuficiencia cardiaca, estenosis aórtica e insuficiencias mitral y aórtica) con la intención de fortalecer la confianza del clínico al tomar en cuenta sus hallazgos.

La eficacia de los signos clínicos se expresará como índices de verosimilitud positivo y negativo (+LR y $-L R)$, que a su vez expresan la magnitud con la que el resultado de una prueba diagnóstica (como una maniobra de examen físico) cambia la probabilidad de que una condición exista ${ }^{4}$. Se incluirán los intervalos de confianza entre corchetes, «[]», siempre que se encuentren disponibles en la fuente original.

Existen ciertas particularidades a considerar con relación a la metodología de la evidencia citada en esta revisión. Algunos puntos específicos incluyen la pobre estandarización de la técnica y entrenamiento entre observadores y 
la comparación directa de un solo signo con los resultados de una tecnología determinada (en lugar de comparar una «impresión clínica completa» con los resultados de varias pruebas). Por tanto, deberá tomarse en cuenta que la mayoría de los estudios citados expresan la eficacia de signos aislados, cuando la práctica clínica se basa en la interpretación de múltiples hallazgos, incluyendo datos obtenidos del examen físico pero también de la historia clínica y el interrogatorio.

El lector deberá tomar en cuenta, además, que la presencia de signos físicos (los auscultatorios, principalmente) es especialmente sensible a los cambios hemodinámicos que suceden continuamente en el aparato cardiovascular la enfermedad. Así, la intensidad de un soplo, por ejemplo, puede cambiar o incluso desaparecer del examen físico del enfermo durante un lapso de algunas pocas horas.

Igualmente, los signos aquí descritos pueden verse exacerbados, atenuados, modificados o abolidos ante la coexistencia de otras condiciones hemodinámicamente significativas (por ejemplo, en el enfermo con enfermedad mitral y aórtica por cardiopatía reumática). La gran mayoría de los estudios aquí presentados han sido realizados en pacientes con enfermedades en su forma «pura» o «casi pura», y el comportamiento e interpretación de los hallazgos puede cambiar radicalmente ante la presencia de comorbilidades (tanto cardiacas como extracardiacas).

Finalmente, la descripción de los procedimientos y las maniobras mencionadas en esta revisión están fuera del objetivo del presente artículo. El lector interesado podrá encontrar una gran variedad de fuentes que detallan con profundidad este tema.

La insuficiencia cardiaca se caracteriza por una serie de hallazgos típicos, incluyendo anomalías en el pulso venoso y arterial, signos de retención de sodio y agua, y una gran cantidad de hallazgos en el examen físico del precordio ${ }^{5}$. Múltiples estudios han reportado el valor del examen físico en el diagnóstico de insuficiencia cardiaca ${ }^{6}$.

El signo que consistentemente ha mostrado el mayor valor clínico es la presencia de un tercer ruido (S3). Datos que se desprenden de 8 ensayos clínicos mostraron un alto índice de verosimilitud positivo (+LR: 11 [4.9-25]) para el diagnóstico de insuficiencia cardiaca, con un índice de verosimilitud negativo relativamente pobre (-LR: 0.88 [0.83-0.94] $)^{6}$. En pacientes con insuficiencia cardiaca aguda, la presencia de un S3 tiene implicaciones pronósticas: en un estudio de 1,281 pacientes hospitalizados por insuficiencia cardiaca aguda, el S3 fue el único signo asociado con un incremento en la mortalidad intrahospitalaria por todas las causas y mortalidad cardiaca tras el análisis multivariado ${ }^{7}$.

La presencia de un S3 también se ha correlacionado con un diámetro telesistólico mayor (+LR: 4.3 [2.7-7.4]). En pacientes con disnea y una historia clínica de enfermedad pulmonar previa (asma, EPOC), un S3 muestra un valor muy alto para el diagnóstico de insuficiencia cardiaca: (+LR: 52 [7.6-425.0]). En este grupo de pacientes, otros signos (como edema pretibial, estertores, sibilancias o congestión hepática) tienen pobre valor para confirmar o descartar insuficiencia cardiaca ${ }^{8}$.

La presencia de un ápex desplazado fuera de la línea medioclavicular muestra una buena correlación positiva con el diagnóstico de insuficiencia cardiaca (+LR: 16 [8.230.9]), sin embargo, la variabilidad interobservador de este hallazgo es considerable.

El análisis de la presión venosa yugular (ingurgitación yugular) también revela información importante: la ingurgitación yugular a más de $3 \mathrm{~cm}$ sobre el ángulo esternal se ha asociado a presiones hemodinámicas elevadas (+LR: 9.7) ${ }^{9}$ y a insuficiencia cardiaca (+LR: 5.1 [3.2-7.9]) ${ }^{6}$. Sin embargo, su asociación con un incremento en la presión telediastólica del VI es pobre: (+LR 3.9) ${ }^{9}$.

Por último, si bien el edema periférico y los estertores crepitantes son signos tradicionales de retención de agua y sodio, su valor clínico en el diagnóstico de insuficiencia cardiaca resulta limitado. Los datos acumulados de 8 estudios clínicos $^{10}$ demuestran una sensibilidad y especificidad del 60 y 78\% para la presencia de estertores crepitantes en el diagnóstico de insuficiencia cardiaca, con índices de verosimilitud pobres: +LR: 2.8 [1.9-4.1] y -LR: 0.51 [0.37-0.70]. De igual forma, el edema periférico tiene una sensibilidad del $50 \%$, una especificidad del $78 \%$ e índices de verosimilitud +LR: 2.3[(1.5-3.7] y -LR: 0.64 [0.47-0.87]. Por tanto, a pesar de ser usados de forma rutinaria como signos de escrutinio de insuficiencia cardiaca, la presencia aislada de estertores crepitantes o de edema pretibial no es suficiente para diagnosticar o descartar el diagnóstico de insuficiencia cardiaca por sí sola. Estos datos deben ser tomados en cuenta con cautela, pues la congestión pulmonar y periférica son datos clínicos que ayudan a valorar la respuesta a tratamiento diurético y el pobre valor estadístico puede venir dado de la amplia gama de causas y condiciones que dinámicamente modifican el estado de volemia y permeabilidad capilar. La figura 1) resume los signos físicos con mayor valor clínico en insuficiencia cardiaca.

En la estenosis aórtica el soplo sistólico crescendodecrescendo sobre el foco aórtico, con irradiación al cuello y carótidas, es el principal componente del examen físico en los pacientes con estenosis aórtica. Efectivamente, la ausencia de soplo en el área aórtica tiene un muy alto valor clínico para descartar estenosis aórtica significativa (-LR: 0.1 [0.02-0.44]), con un valor predictivo negativo del 99.6\% ${ }^{11}$.

Por el contrario, la presencia de un soplo sistólico en el área aórtica puede tener otros orígenes (estenosis pulmonar, comunicación interventricular, estenosis aórtica subvalvular, insuficiencia mitral, etc.). En estos casos, existen varios

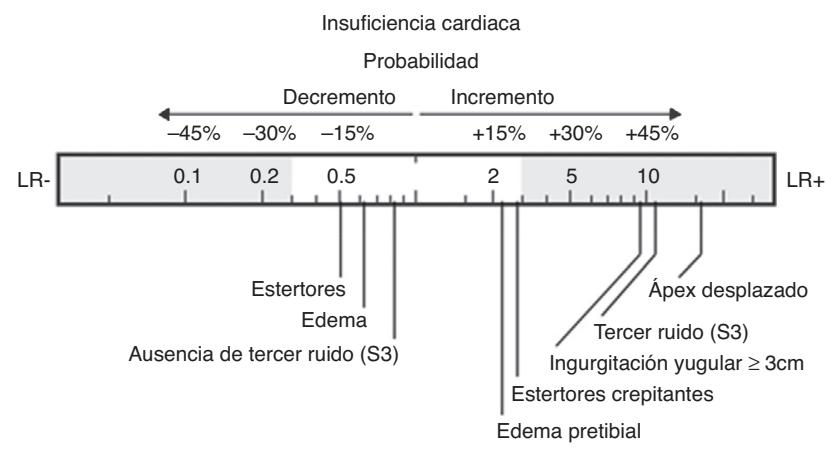

Figura 1 Signos con mejor rendimiento diagnóstico para descartar (-LR, a la izquierda) o confirmar (+LR, a la derecha) el diagnóstico de insuficiencia cardiaca. 
datos clínicos que pueden contribuir a diferenciar el origen aórtico de la lesión: la radiación del soplo a la región carótida (+LR: 8.1 [4-16]), la presencia de un ascenso retrasado en el pulso carotídeo (pulsus tardus) (+LR: 6.8 [4-11.5]), la ausencia 0 intensidad disminuida del segundo ruido (+LR: $7.5[3.2-17])$ y el volumen o intensidad reducido del pulso carotídeo (pulsus parvus) (+LR: 2 [1-3.2]). Si bien de forma aislada ninguno de estos signos puede confirmar por sí solo la presencia de estenosis aórtica, la combinación de 3 o 4 de ellos tiene un muy alto valor diagnóstico positivo (+LR: $40[6.6-240])^{11}$.

La intensidad del soplo es una característica que contribuye a diferenciar la severidad de la condición: en un estudio de 123 pacientes examinados por un único cardiólogo, un soplo grado $3 / 6$ predijo un gradiente pico mayor de $64 \mathrm{mmHg}$ con una alta especificidad (90\%), pero con baja sensibilidad $(29 \%)^{12}$. A la par, la intensidad del componente aórtico del segundo ruido ha sido descrito como un parámetro de severidad: cuando se encuentra ausente, traduce importante calcificación y limitación de la movilidad. Sin embargo, en una serie consecutiva de pacientes, la intensidad del segundo ruido no fue capaz de distinguir las formas severas de las leve a moderadas ${ }^{13}$.

Finalmente, en cuanto a implicaciones pronósticas del examen físico en estenosis aórtica, el trabajo de Munt et al. ${ }^{12}$ tuvo como objetivo correlacionar los resultados del examen físico con desenlaces clínicos como cambio valvular o muerte. El estudio mostró que tras el análisis de regresión multivariada de Cox en 123 pacientes con estenosis aórtica severa, el único signo que se asoció a peor pronóstico fue un pulso carotídeo disminuido (pulsus parvus): la supervivencia a 2 años fue del $89 \%$ en aquellos con pulso normal vs. el $65 \%$ en aquellos con pulso severamente disminuido. Estos resultados deben tomarse con cautela por las limitaciones del estudio. La figura 2 resume los signos físicos con mayor valor clínico en estenosis aórtica.

En la insuficiencia mitral el soplo sistólico regurgitante con carácter de «chorro de vapor» se ha descrito como el hallazgo central de la insuficiencia mitral. Un estudio realizado por Attenhofer et al. mostró que la sensibilidad del examen físico cardiovascular para detectar insuficiencia mitral fue aceptable: aproximadamente del $88 \%$. No existe evidencia con relación a que el tono o carácter del soplo se asocien al origen mitral de la lesión; sin embargo, un soplo sistólico auscultado sobre el ápex que se extiende a

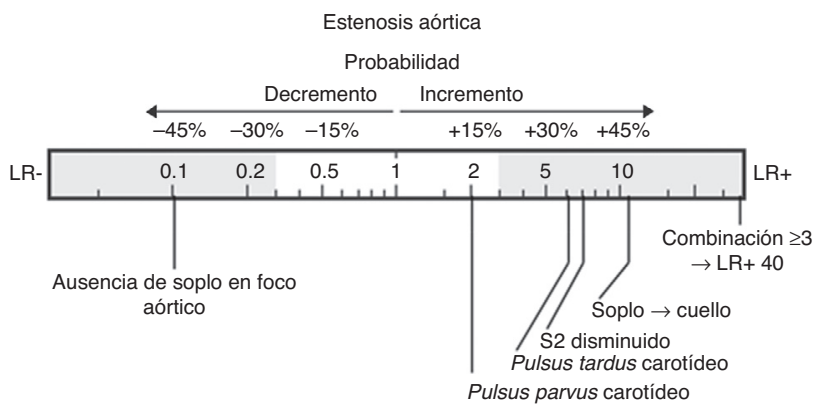

Figura 2 Signos con mejor rendimiento diagnóstico para descartar (-LR, a la izquierda) o confirmar (+LR, a la derecha) el diagnóstico de estenosis aórtica. al menos la línea axilar anterior indica insuficiencia mitral crónica moderada o mayor con un valor clínico moderado (+LR: 6.8 [3.9-11.9]) $)^{14}$. Por el contrario, es importante enfatizar que tanto en la insuficiencia mitral aguda postinfarto como en la insuficiencia mitral crónica, la ausencia de soplo sistólico no permite excluir el diagnóstico (-LR: 0.66 $[0.25-1])^{15}$.

La eficacia del examen físico en insuficiencia mitral no es un valor absoluto, sino que va con relación a la severidad. Al incrementar la gravedad de la lesión, también aumenta la posibilidad de encontrar un soplo: de un $28 \%$ en formas leves hasta un $100 \%$ en formas graves ${ }^{16}$.

La palpación del ápex tiene importancia en la insuficiencia mitral; especialmente en el grupo de pacientes que presentan datos de estenosis mitral; el hallazgo de un impulso apical hipercinético es un hallazgo que traduce la presencia de una segunda lesión, frecuentemente insuficiencia mitral o insuficiencia aórtica, con un buen valor clínico (+LR: 11.2$)^{17}$.

La intensidad del soplo muestra buena correlación con la severidad de la regurgitación: soplos muy fuertes (grados 4-5/6) incrementan la posibilidad de insuficiencia mitral severa de forma substancial (+LR: 14 [3.3-56]), mientras que grados 1-2/6 disminuyen considerablemente la posibilidad de lesión severa (-LR: 0.19 [0.11-0.33] $)^{15}$.

Finalmente, la presencia de un segundo ruido fuerte tiene un moderado valor clínico para detectar insuficiencia mitral en pacientes con soplo sistólico compatible (+LR: 4.7) ${ }^{16}$. Por el contrario, la presencia de un S3 se ha considerado como un marcador de severidad en insuficiencia mitral, pero los resultados en cuanto a la correlación del S3 y un incremento en las presiones de llenado son contradictorios ${ }^{18}$. La figura 3) resume los signos físicos con mayor valor clínico en insuficiencia mitral.

En la insuficiencia aórtica la palpación del choque de la punta brinda información útil en el paciente con insuficiencia aórtica. Si bien la presencia de un impulso apical sostenido tiene poco valor al momento de detectar insuficiencia severa (+LR: 2.4), la ausencia de un impulso apical sostenido o un choque de la punta normal disminuyen significativamente la posibilidad de insuficiencia moderada a severa (-LR: 0.1$)^{19}$. La presencia de un S3 se ha asociado insuficiencia severa (+LR: 5.9) y a una fracción de expulsión del ventrículo izquierdo menor del 50\% (+LR: 8.3) con un valor clínico moderado ${ }^{18}$.

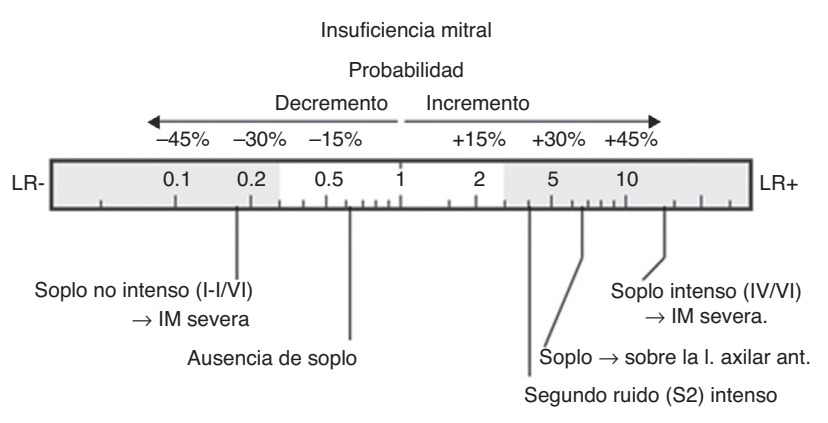

Figura 3 Signos con mejor rendimiento diagnóstico para descartar (-LR, a la izquierda) o confirmar (+LR, a la derecha) el diagnóstico de insuficiencia mitral. 


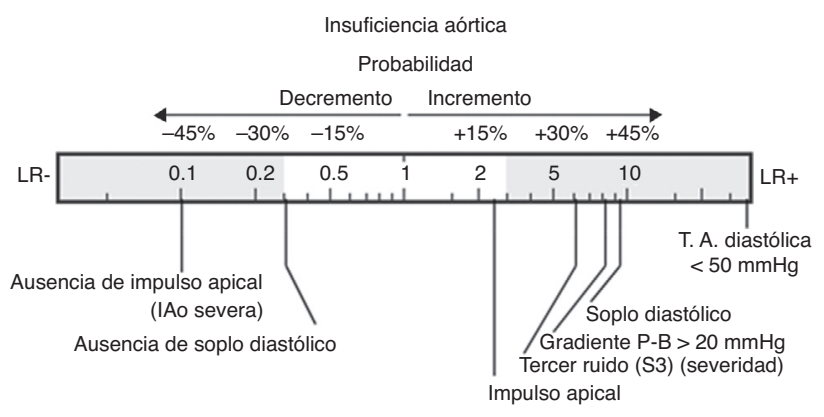

Figura 4 Signos con mejor rendimiento diagnóstico para descartar (-LR, a la izquierda) o confirmar (+LR, a la derecha) el diagnóstico de insuficiencia aórtica.

El soplo diastólico temprano sobre la columna aórtica tiene un rendimiento diagnóstico considerable (+LR: 9.9) ${ }^{20}$ para detectar insuficiencia aórtica más que ligera. La ausencia de soplo diastólico tiene también un valor clínico moderado (-LR: 0.3 ) para descartar insuficiencia aórtica, especialmente insuficiencia aórtica moderada a severa (-LR: 0.1$)^{21}$. Auscultar el soplo diastólico temprano mejor en el lado derecho del esternón es un signo que puede indicar, con un valor clínico moderado (+LR 8.2), la dilatación de la raíz aórtica ${ }^{22}$.

La presión arterial, en específico las cifras de presión arterial diastólica pueden ser de gran ayuda para detectar formas moderadas-severas de insuficiencia aórtica. Una presión diastólica menor de $50 \mathrm{mmHg}$ tiene un alto valor clínico (+LR 19.3), aunque con baja sensibilidad (30-50\%), para detectar insuficiencia severa ${ }^{23}$.

Finalmente, la presencia de un gradiente poplíteobraquial mayor de $20 \mathrm{mmHg}$ muestra un valor clínico moderado (+LR: 8.2 [1.5-78]) para insuficiencia aórtica más que ligera. En este mismo estudio, otros signos periféricos no mostraron utilidad para diagnosticar o descartar insuficiencia aórtica ${ }^{21}$. La figura 4) resume los signos físicos con mayor valor clínico en insuficiencia aórtica.

\section{Conclusiones}

El examen físico cardiovascular continúa siendo una herramienta útil, basada en evidencias, que ofrece información válida con relación a condiciones clínicas comunes como la insuficiencia cardiaca, la estenosis aórtica, y las insuficiencias mitral y aórtica. Mayoritariamente, los signos físicos tienen buen valor clínico positivo (índice de verosimilitud positivo) y carecen de valor clínico negativo. La investigación sobre el examen físico cardiovascular es un área abierta que podrá complementar el quehacer diario del cardiólogo.

\section{Financiación}

No se recibió patrocinio de ningún tipo para llevar a cabo este artículo

\section{Conflicto de intereses}

Los autores declaran no tener ningún conflicto de intereses.

\section{Bibliografía}

1. Bailey CP. The surgical treatment of mitral stenosis (mitral commissurotomy). Dis Chest. 1949;15:377-97.

2. Tavel ME. A glorious past-but does it have a future? Circulation. 1996;93:1250-3.

3. Fred HL. Hyposkillia. Texas Heart Inst J. 2005;32:255-7.

4. Pauker SG, Kassirer JP. Therapeutic decision making: A costbenefit analysis. N Engl J Med. 1975;293:229-34.

5. Guadalajara JF. Historia clínica. En: Cardiología. Séptima edición Ciudad de México: Méndez editores; 2012.

6. Leier CV, Chatterjee K. The physical examination in heart failure-Part I. Congest Heart Fail 2007;13:41-7.

7. Minami Y, Kajimoto K, Sato N, et al. Third heart sound in hospitalised patients with acute heart failure: Insights from the ATTEND study. Int J Clin Pract. 2015;69:820-8.

8. McCullough PA, Hollander JE, Nowak RM, et al. Uncovering heart failure in patients with a history of pulmonary disease: Rationale for the early use of B-type natriuretic peptide in the emergency department. Acad Emerg Med. 2003;10: 198-204.

9. Butman SM, Ewy GA, Standen JR, et al. Bedside cardiovascular examination in patients with severe chronic heart failure: Importance of rest or inducible jugular venous distension. J Am Coll Cardiol. 1993;22:968-74.

10. Wang CS, FitzGerald JM, Schulzer M, et al. Does this dyspneic patient in the emergency department have congestive heart failure. JAMA. 2005;294:1944-56.

11. Etchells E, Glenns V, Shadowitz S, et al. A bedside clinical prediction rule for detecting moderate or severe aortic stenosis. Gen Intern Med. 1998;13:699-704.

12. Munt $\mathrm{BI}$, Legget $\mathrm{ME}$, Kraft $\mathrm{CD}$, et al. Physical examination in valvular aortic stenosis: Correlation with stenosis severity and prediction of clinical outcome. Am Heart J. 1999;137: 298-306.

13. Aronow WS, Kronzon I. Prevalence and severity of valvular aortic stenosis determined by Doppler echocardiography and its association with echocardiographic and electrocardiographic left ventricular hypertrophy and physical signs of aortic stenosis in elderly patients. Am J Cardiol. 1991;67:776-7.

14. Attenhofer Jost CH, Turina J, Mayer K, et al. Echocardiography in the evaluation of systolic murmurs of unknown cause. Am J Med. 2000;108:614-20.

15. Panidis IP, McAllister M, Ross J, et al. Prevalence and severity of mitral regurgitation in the mitral valve prolapse syndrome: A Doppler echocardiographic study of 80 patients. J Am Coll Cardiol. 1986;7:975-81.

16. McGee S. Etiology and diagnosis of systolic murmurs in adults. Am J Med. 2010;123:913-21.

17. Wood P. An appreciation of mitral stenosis: Part 1. Clinical features. Part 2. Investigations and results. $\mathrm{Br}$ Med J. 1954;1:1051-63.

18. Tribouilloy CM, Enriquez-Sarano M, Mohty D, et al. Pathophysiologic determinants of third heart sounds: A prospective clinical and Doppler echocardiographic study. Am J Med. 2001;111:96-102.

19. Frank MJ, Casanegra P, Migliori AJ, et al. The clinical evaluation of aortic regurgitation. Arch Intern Med. 1965;116:357-65.

20. Rahko PS. Prevalence of regurgitant murmurs in patients with valvular regurgitation detected by Doppler echocardiography. Ann Intern Med. 1989;111:466-72. 
21. Grayburn PA, Smith MD, Handshoe R, et al. Detection of aortic insufficiency by standard echocardiography, pulsed Doppler echocardiography, and auscultation: A comparison of accuracies. Ann Intern Med. 1986;104:599-605.

22. Sakamoto T, Kawai N, Uozumi A, et al. The point of maximum intensity of aortic diastolic regurgitant murmur, with special emphasis to the right-sided aortic diastolic murmur. Jap Heart J. 1968;9:117-33.

23. Linhart JW. Aortic regurgitation: Clinical, hemodynamic, surgical, and angiographic correlations. Ann Thorac Surg. 1971;11:27-37. 